\title{
Using Alnuset to construct the notions of equivalence and equality in algebra
}

\author{
Giampaolo Chiappini, Bettina Pedemonte and Elisabetta Robotti \\ Istituto per le Tecnologie Didattiche - CNR \\ V. De Marini 6, 16149 Genova, Italy \\ \{chiappini, pedemonte, robotti\}@itd.cnr.it \\ http://www.itd.cnr.it
}

\begin{abstract}
We analyse the role of technology in the development of algebraic crucial knowledge and competences, such as those involved in the comprehension and use of the notions of equivalence and equality. The hypothesis of our research is that technology can be exploited to make available new operative and representative possibilities which structure a new phenomenological space to investigate the algebraic knowledge and to improve the teaching and learning processes of algebra. We analyse the characteristics of the phenomenological space structured by Alnuset, a system developed by the authors within ReMath European project. Through examples taken from experimentations that we have performed with Alnuset, we illustrate and discuss how such characteristics can be exploited to develop competences and knowledge in the algebraic domain and to improve the teaching of algebra.
\end{abstract}

\section{Introduction}

What does it mean that two algebraic expressions are equivalent? How can the equal sign between two algebraic expressions be interpreted? Can an algebraic expression be equal to another one without being equivalent to it? What is the role of the universal quantifier and the existential quantifier in characterising different meanings that the equal sign can assume in a proposition? How can the transformation of an algebraic expression and the solution of an equation be interpreted?

Answering these questions might not be easy for students, as well as justifying appropriately their replies or fulfilling tasks that require a good mastery of the notions of equivalence and equality involved in these questions [1,2]. In the current teaching practice, students mainly learn to use algebraic rules properly, but they often show difficulties both in justifying them and in assigning meanings to the previously mentioned notions. In order to develop these competencies changes and new levels of mediation in teaching are necessary. A first essential change might 
consist in focusing the students' attention either on the numerical quantities indicated by a literal symbol or expression or on the numerical quantities that can condition the truth value of an equality. We name this kind of algebra as algebra of quantities. We observe that currently it could be hard to work out this change because available tools are not quite suitable to mediate the relationship between algebraic expressions and equalities on one hand, and numerical quantities on the other. Moreover, the current approach to algebra teaching based on formal operations should be deeply modified and integrated with algebra of quantities. The main purpose of such change is the modification of current algebraic manipulation teaching, mainly centred on learning and reinforcement of algebraic transformation rules through a drill and practice pedagogical strategy. We think that the change should favour both the solution of cognitive problems involved in the construction of schemes for algebraic transformations and the development of semiosis processes to assign meaning to the performed transformations and to the involved algebraic phenomena. In order to work out these changes and to integrate algebra of quantities with algebra of formal operations, specific teaching mediation tools are necessary. We have designed these tools and we have embedded and integrated them in the Alnuset system. In this workshop we discuss the role of Alnuset in structuring a new phenomenological space to favour the development of algebraic notions of equivalence and equality.

\section{The Alnuset system}

The Alnuset system (Algebra of NUmerical SETs) has been developed within the ReMath European project (IST-4 -26751) to allow the development of algebra of quantities in school practice and its integration with a deeply innovated teaching of algebra of formal operations. This system is oriented to students of lower and upper secondary school (from 13 to $17 \mathrm{yrs}$ ). Alnuset includes three closely integrated components - the Algebraic Line component, the Symbolic Manipulator component and the Cartesian Plan component. In this workshop we refer to the first two components designed to favour the integrated development of algebra of quantities and algebra of formal operations respectively.

The Algebraic Line Component

The Algebraic Line structures a new phenomenological space where processes, relationships and objects of algebraic nature can be investigated through a quantitative approach. Through digital technology the traditional numbers line was characterized with new operative and representative possibilities, namely:

- with three different editors to construct and represent algebraic expressions on the line, specifically with a geometrical editor, a linear editor and a bidimensional editor;

- with the drag of mobile points associated to letters on the line which determines the automatic movement of points of expressions containing the dragged letters on the same line;

- with a graphic and computational model to determine the roots of polynomials;

- with graphical models to define and validate the truth set of algebraic propositions. 
The user can directly and dynamically control these new operative and representative possibilities exploiting their visual, spatial and motor experience. These possibilities can be used to explore what expressions and propositions denote within the considered numerical domain.

\section{The Symbolic Manipulator Component}

The Symbolic Manipulator structures a new phenomenological space where the norms, the rules and the conventions of algebra can be investigated in order to structure an idea of Algebra as the science of formal operations, namely, as the theory and practice of formal operations that preserve equivalence in the performed transformations. This space is characterized by new operative and representative possibilities such as:

- exploring the hierarchical structure of the expression or proposition that has to be manipulated;

- exploring the rules of transformation of the interface that can be applied on each selected structure;

- exploring the effects that the applications of a rule produce;

- verifying that in the performed transformation the equivalence has been preserved;

- creating a new rule of transformation, once it has been proved using the rules available with the interface;

These new operative and representative possibilities can be exploited to construct general schemes for algebraic transformations and to develop a theoretical approach to algebraic transformation that can be accessible to students.

\section{The construction of meanings for the notion of equality and of equivalence in algebra}

In order to discuss the usefulness of the phenomenological space structured by the Algebraic Line of Alnuset as referred to the development of the notion of algebraic equality, let us present, as an example, the following task taken from our experimentation.

Consider the two following equalities $2 * x+3 * x=5 * x$ e $2 * x+3=5 * x$. Write how you can interpret them Represent the expressions $2^{*} x+3,2 * x+3 * x$ e $5^{*} x$ on the algebraic line of Alnuset and use the editor to construct the two equalities. Exploit the representative possibilities of Alnuset to verify the previously given answer and to eventually modify it.

Many students of the 9-10 grade interpret the first equality as correct and the second one as wrong; few students are able to interpret them respectively as identity and as equation. In the activity with the algebraic line of Alnuset some representative phenomena useful to favour the development the understanding of algebraic equality can emerge. The first phenomenon concerns the representation of the expressions on the algebraic line (see Figure 1.1 and Figure 1.2). Every point on the line is characterized by a post-it that contains all the constructed expressions which indicate the value of that point. We observe that the two expressions $2 x+3 x$ and $5 x$ correspond to a same point on the algebraic line and they always belong to the same 
post-it when the mobile point associated to the variable $\mathrm{x}$ is dragged along the line (Figure 1.1). The expression $2 x+3$ belongs to the same post-it of $5^{*} x$ only when the mobile point of the variable $x$ is dragged on the numeric value 1 of the line (Figure 1.2). The drag of the mobile point associated to the variable $x$ can be a useful way to experience what the expressions indicate, while the post-it can be a useful representation to explore the value of variables that make their result equal.

A second important representative phenomenon emerges when the two equalities $2 x+3 x=5 x$ and $2 x+3=5 x$ are inserted in the Truth Sets window of the algebraic line environment through the algebraic editor (see Figure 1.3 and Figure 1.4)

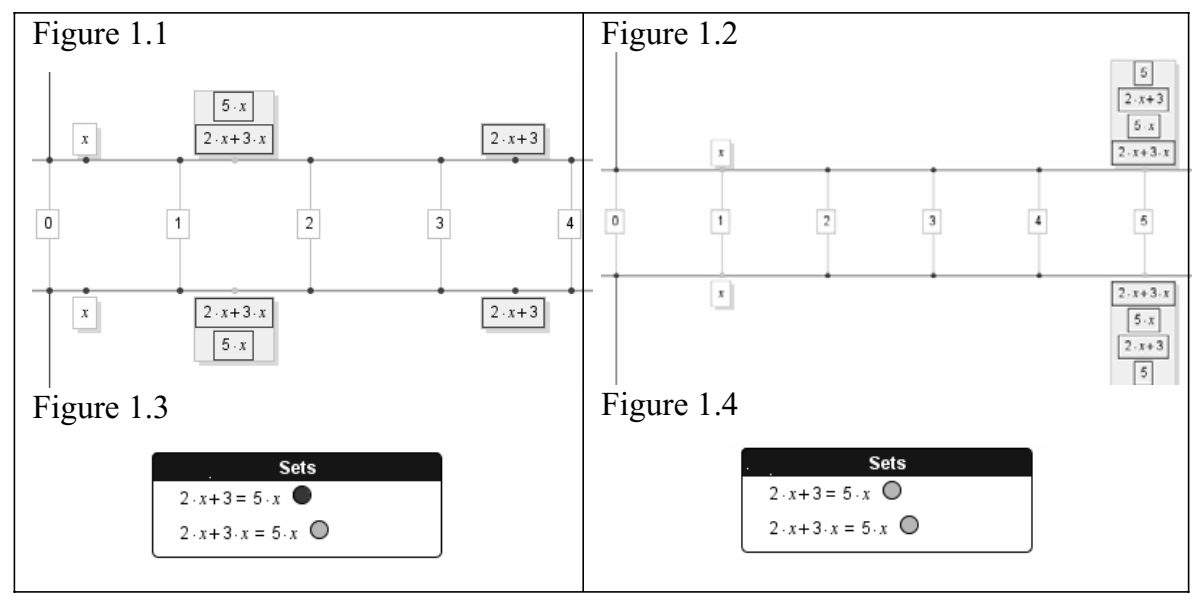

Fig. 1. The representation of the expressions $5 x, 2 x+3 x, 2 x+3$ on the algebraic line

In the Truth Sets window the two equalities are associated to a ball that turns red or green according to the truth value of these equalities (respectively false and true). The truth value of these equalities and of their corresponding balls can be conditioned by the movement of the variable point $\mathrm{x}$ on the algebraic line. In our example, when the mobile point $\mathrm{x}$ is dragged on the value 1 , both the balls of the two equalities are green (Figure 1.4), while when it is dragged on any another value of the line the ball corresponding to the equation $2 x+3=5 x$ is red and the ball corresponding to the identity $2 x+3 x=5 x$ remains green (Figure 1.3).

\section{References}

1. Arzarello F., Bazzini L., Chiappini G., (2001), A model for analyzing algebraic process of thinking, in Sutherland R., Rojano T., Bell A. (Eds), Perspectives on school algebra, Kluwer Academic Publisher, p. 61-82.

2. Sfard, A., Linchevski, L. (1992) Equations and inequalities: processes without objects? In Geeslin W. \& Ferrini-Mundy J. (Ed.), Proceedings of the Sixteenth Annual Conference of the International Group for the Psychology of Mathematics Education PMEXVI, 3, Durham, NH: University of New Hampshire, p.136. 